\title{
A method for culturing Gram-negative skin microbiota
}

\author{
Ian A. Myles ${ }^{1 *}$, Jensen D. Reckhow ${ }^{1 \dagger}$, Kelli W. Williams ${ }^{1 \dagger}$, Inka Sastalla ${ }^{1}$, Karen M. Frank ${ }^{2}$ and Sandip K. Datta ${ }^{1}$
}

\begin{abstract}
Background: Commensal Gram-negative (CGN) microbiota have been identified on human skin by DNA sequencing; however, methods to reliably culture viable Gram-negative skin organisms have not been previously described.

Results: Through the use of selective antibiotics and minimal media we developed methods to culture CGN from skin swabs. We identified several previously uncharacterized CGN at the species level by optimizing growth conditions and limiting the inhibitory effects of nutrient shock, temperature, and bacterial competition, factors that may have previously limited CGN isolation from skin cultures.

Conclusions: Our protocol will permit future functional studies on the influences of CGN on skin homeostasis and disease.
\end{abstract}

Keywords: Bacteriology, Skin, Microbiome, Culture techniques

\section{Background}

A wealth of recent work has identified the microbiome as a major influence on human health and disease. Topographical surveys of the skin microbiota by $16 \mathrm{~S}$ ribosomal RNA gene [1] and metagenomic shotgun [2] sequencing have highlighted the bacterial diversity found on the human body. These studies confirmed the previously appreciated prevalence of Gram-positive bacteria on the skin, including various Staphylococcus species and Actinobacteria such as Propionibacterium and Corynebacterium. Historically, low and inconsistent yields of Gram-negative bacteria from skin by culture led to the conclusion that Gram-negative species were absent or transient inhabitants of human skin [3, 4]. However, genomic approaches have identified Gram-negative bacteria as significant constituents of the skin biome, particularly at sites such as the antecubital fossa and volar forearm [1, 2]. Limitations in DNA analysis techniques have largely prevented species-level identification of these Gram-negative bacteria in the skin microbiome $[1,2,5]$. Here we describe novel methods to culture

\footnotetext{
*Correspondence: mylesi@niaid.nih.gov

${ }^{\dagger}$ Equal contributors

'Bacterial Pathogenesis Unit, Laboratory of Clinical Infectious Diseases, National Institute of Allergy and Infectious Diseases, National Institutes of Health, Bethesda, MD, USA

Full list of author information is available at the end of the article
}

viable commensal Gram-negative (CGN) skin bacteria. This will allow species-level identification, whole genome sequencing, design of gene primers for enhanced molecular identification, and functional characterization of these bacteria and their role in skin homeostasis and disease.

\section{Methods}

\section{Subject selection and sampling}

Thirteen healthy adults, with no history of skin disease, were seen in our outpatient clinic. The participants were asked to refrain from bathing for the $24 \mathrm{~h}$ prior to their visits. For the nine participants who were amenable and able to return for repeat visits, isolation procedures were repeated to assess temporal consistency of culture findings. The antecubital fossa and volar forearm were selected as culture sites due to their propensity to contain CGN in published microbiome studies [1,2] and their relevance as medically important sites for skin conditions such as atopic dermatitis.

\section{Gram-negative bacterial isolation}

We first moistened two FloqSwabs (Copan, Brescia, Italy) in sterile phosphate buffered saline (PBS; Corning Cellgro, Corning, NY). Both swabs were simultaneously rubbed on the subject's skin at the antecubital fossa and volar forearm vigorously for 15-30 s. One swab was placed into a $15 \mathrm{~mL}$ conical 
tube (Corning Life, Corning, NY) with $2 \mathrm{~mL}$ of sterile Hank's balanced salt solution (HBSS; SigmaAldrich), vancomycin $(300 \mathrm{ug} / \mathrm{mL})$, and amphotericin B (5ug/mL; Sigma-Aldrich, St. Louis, MO) to inhibit growth of Gram-positive bacteria and fungi. The remaining swab was placed into a $15 \mathrm{~mL}$ conical tube containing $2 \mathrm{~mL}$ of R2A broth (Teknova, Hollister, CA) with similar concentrations of vancomycin and amphotericin $\mathrm{B}$. The tubes, with swabs left in place, were then incubated at $32{ }^{\circ} \mathrm{C}$ with constant shaking for $48-72 \mathrm{~h}$ under aerobic conditions before plating $100 \mathrm{uL}$ from each tube onto an R2A (Reasoner's 2A) agar plate (Remel, Lenexa, KS). R2A media is a relatively nutrient poor agar typically used for the isolation of slow-growing bacteria in potable water [6] (Additional file 1: Supplemental methods). Colonies were appreciable $48-72 \mathrm{~h}$ later. Individual colonies were then taken for species identification by mass spectrometry using matrix-assisted laser desorption/ionization-time of flight (MALDI-TOF) analysis. Bacterial protein extraction for MALDI-TOF MS using the BioTyper (v3.1, Bruker Daltonics Inc., Billerica, MA) was performed using previously described methods [7], instrument settings and calibration $[8,9]$. BioTyper identification was supplemented by additional mass spectra profiles provided by several NIH developed databases $[7,10,11]$. Nine of the participants were swabbed at three different times, separated by at least 3 months over the course of a year and identical species were isolated on these sequential cultures (participants 1-2, 5-9, 12-13).

\section{Gram-positive bacterial isolation}

Skin swabs obtained as described above were plated directly on blood agar or brain heart infusion agar (BHI) and incubated under aerobic conditions at $37^{\circ} \mathrm{C}$. Staphylococcus aureus was initially distinguished from other staphylococcal species by mannitol fermentation on mannitol salt agar. Speciation of suspected S. aureus isolates were confirmed by measuring coagulase activity (Fluka Chemicals, Switzerland).

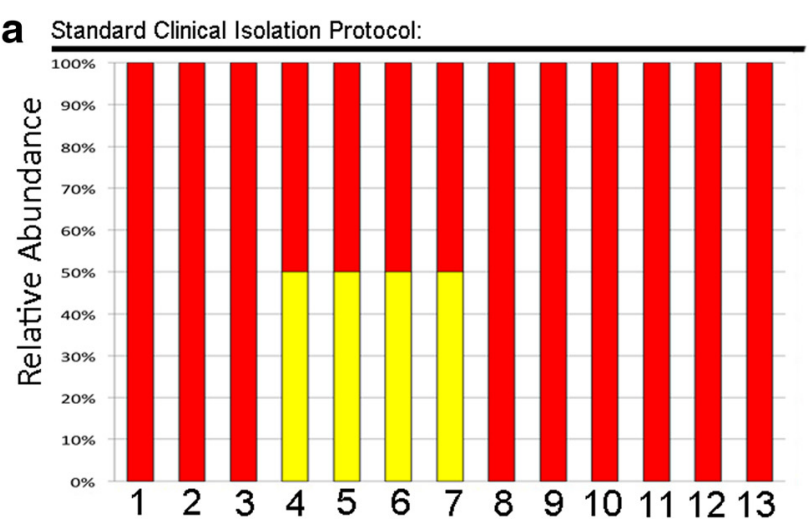

b Modified Clinical Isolation Protocol:

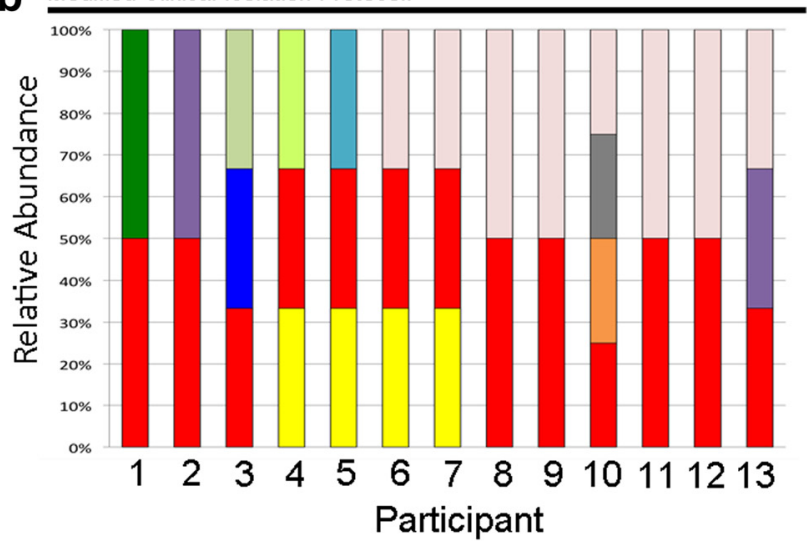

\author{
$\square$ Roseomonas mucosa \\ - Rhodotorula spp. \\ $\square$ Moraxella osloensis \\ $\square$ Methylobacteria spp. \\ $\square$ Pantoea septica \\ $\square$ Pseudomonas oryzihabitans \\ - Pseudomonas aeruginosa \\ $\square$ Pseudomonas luteola \\ - Acinetobacter radioresistens \\ - Staphylococcus spp. (non-aureus) \\ $\square$ Staphylococcus aureus
}

Fig. 1 Culture protocol modifications allow isolation of Gram-negative skin microbiota. a Thirteen participants underwent standard clinical skin bacteria isolation using swabs plated onto blood, mannitol salt, $\mathrm{BHI}$, chocolate, and MacConkey agar incubated at $37^{\circ} \mathrm{C}$. $\mathbf{b}$ The same participants underwent modified bacterial isolation as described. The relative abundance of cultured bacterial isolates from each participant is shown on the vertical axis. All bacteria were identified by MALDI-TOF mass spectrometry analysis, except Staphylococcus species were identified by characteristic growth on mannitol salt agar plates with positives confirmed by coagulase testing. For participants that were re-sampled, no discrepancies between initial and subsequent isolations were found 


\section{Consent}

Written informed consent was obtained for all participants in this study. All participants were adults.

\section{Results and discussion}

Modification of media and temperature allows isolation of CGN from skin

Standard culture techniques for Gram-negative bacteria from sources other than skin involve incubation at $37^{\circ} \mathrm{C}$ using liquid media such as tryptic soy broth (TSB), or on solid media such as chocolate, blood, or MacConkey [12]. We attempted to culture skin bacteria from thirteen participants by plating forearm swabs onto $5 \%$ sheep blood, mannitol salt, BHI, chocolate, and MacConkey agar incubated at $37^{\circ} \mathrm{C}$. Our use of these techniques readily isolated staphylococcal species from multiple healthy volunteers but failed to culture any Gram-negative isolates, even with the use of a Gram-negative selective agar such as MacConkey (Fig. 1a).

The use of our modified Gram-negative bacteria isolation protocol (see Methods) yielded several Gramnegative species from the forearm skin of healthy volunteers (Fig. 1b). The predominant Gram-negative bacterium isolated by our methods was Roseomonas mucosa, a member of the alphaproteobacteria class. For two volunteers (2 and 3), no growth was seen from R2A broth, but indicated species were isolated from the HBSS tube. Other species isolated included the gammaproteobacteria Pseudomonas aeruginosa, Pseudomonas luteola, Pseudomonas oryzihabitans, Acinetobacter radioresistens, Pantoea septica, and Moraxella osloensis. Swabs from one individual grew Methylobacterium species (alphaproteobacteria). These results are consistent with prior reports using phylogenetic and metagenomic sequence analysis $[1,2]$. Two subjects grew yeast, $R$ hodotorula spp. (R. mucilaginosa and R. minuta/slooffiae), despite the presence of amphotericin B during culture, including one healthy subject from whom no Gramnegatives were cultured (identified by MALDI-TOF followed by sequencing of ITS region as described previously [8]). Colonies of Roseomonas mucosa and both Rhodotorula spp. had some initial morphological similarities that may make discernment difficult if each species is viewed in isolation (Fig. 2), but they were readily distinguished by Gram-staining.

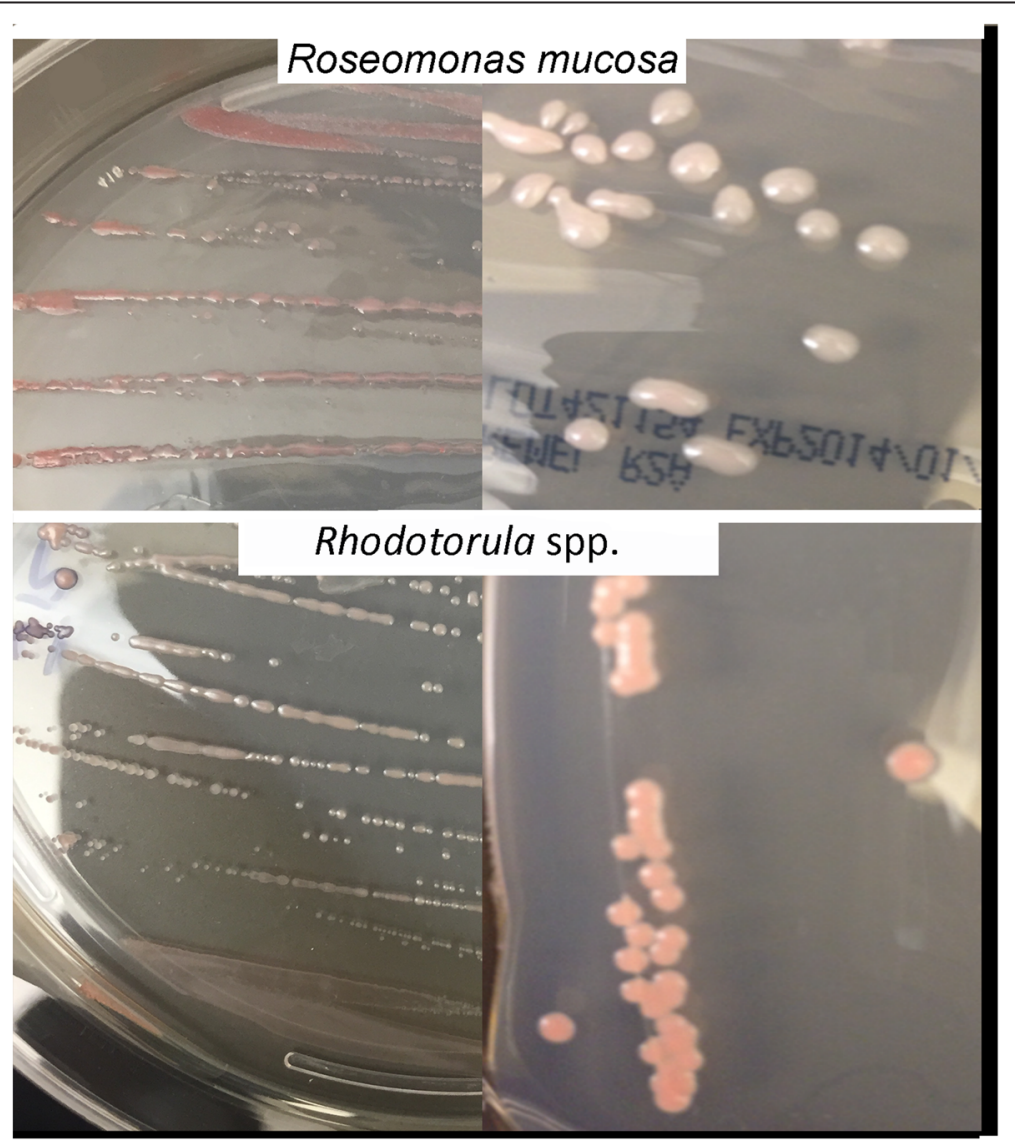

Fig. 2 Colony morphology for Roseomonas mucosa and Rhodotorula spp. Colony morphology for two different strains of Roseomonas mucosa (top) and Rhodotorula spp. (bottom; R. mucilaginosa, right; R. minuta/slooffiae, left) streaked linearly (left) or with four-quadrant technique (right) on R2A agar 
CGN from skin have more protracted growth curves than Gram-positive isolates

The growth kinetics of the CGN isolates were variable, but most showed completion of exponential growth by 4-6 h (Fig. 3a-b). Staphylococcal strains reached a nearly five-log higher CFU total by six hours in either nutrientpoor or nutrient-rich broth (Fig. 3a-b). Thus, one explanation for prior failures to culture CGN could involve the ability of staphylococci and other robustly growing organisms to outpace the growth of CGN, making inclusion of antimicrobials, such as vancomycin and amphotericin B, critical for the isolation of CGN flora.

\section{CGN from skin are slower growing than Gram-positive isolates}

After incubation of skin swabs in HBSS or R2A, the initial isolation of $R$. mucosa, P. septica, and M. osloensis was only successful when using R2A plates (Table 1). Maximal yield was obtained when these isolates were incubated at $32^{\circ} \mathrm{C}$, a temperature more reflective of the

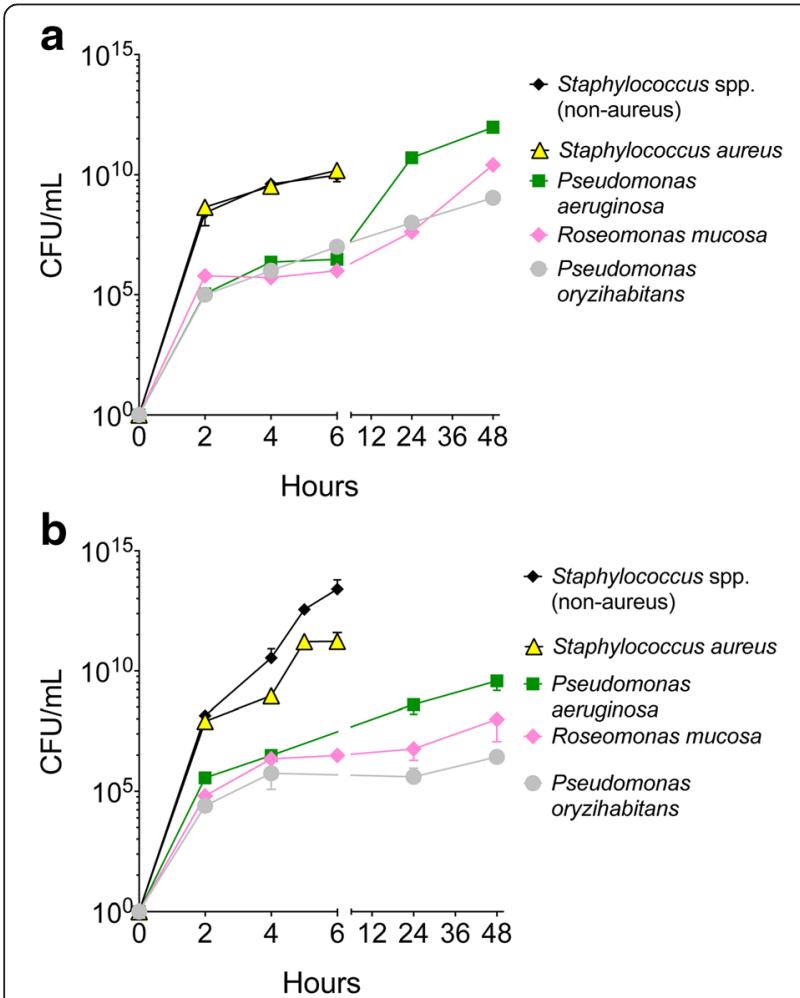

Fig. 3 Growth curves for select commensal Gram-negative and Staphylococcus species. A single colony of bacteria was added to liquid media at time zero for all indicated isolates. Colony forming units (CFU) assessed by serial dilutions at indicated time points. a Growth performed at $32^{\circ} \mathrm{C}$, in R2A broth for both CGN and staphylococcal species for direct comparison of growth kinetics. $\mathbf{b}$ Comparison of optimized growth kinetics; CGN cultured at $32^{\circ} \mathrm{C}$ in R2A broth,

Staphylococcus species cultured at $37^{\circ} \mathrm{C}$ in TSB. Data are representative of two or more independent experiments and depicted as mean + SD. Each species represented by 1-3 clinical isolates skin surface than $37^{\circ} \mathrm{C}$ [13]. Initial isolation of the Pseudomonas species were additionally successful on blood agar if incubated at $32{ }^{\circ} \mathrm{C}$ and on chocolate agar at both $32^{\circ} \mathrm{C}$ and $37^{\circ} \mathrm{C}$ (Table 1). Once our initial swabs yielded viable bacterial colonies, these could then be subsequently grown on many nutrient rich agars (Table 1). However, our $R$. mucosa strains failed to grow on chocolate or MacConkey agar, even when plating at a $1 \mathrm{e} 8$ or greater inoculum. Taken together, these findings suggest that previous failures to consistently isolate CGN bacteria may have been due to staphylococcal competition, the use of nutrient rich media, and choice of $37^{\circ} \mathrm{C}$ incubation. However, once a high enough pure inoculum has been collected, limitations in media and temperature can be overcome on select media.

\section{CGN from the skin showed evidence of nutrient shock}

Failure of bacteria to grow in nutrient rich environments has been termed 'nutrient shock'. This has been proposed as a mechanism to explain the presence of 'viable but nonculturable bacteria' seen by staining techniques, and the detection of DNA signatures for bacteria that are nonculturable. Recent work has quantified evidence of nutrient shock [14]. We performed similar analysis by first growing $R$. mucosa, P. septica, and M. osloensis in R2A broth for $48 \mathrm{~h}$. Then each isolate was diluted 1:50 into either TSB (nutrient rich) or R2A (nutrient poor) and plated on R2A agar immediately and again after $48 \mathrm{~h}$ of incubation at $32^{\circ} \mathrm{C}$. Dilution into TSB led to an $80 \%$ reduction in CFU after $48 \mathrm{~h}$ for $R$. mucosa and $M$. osloensis compared to dilution into R2A (Additional file 1: Figure S1). In contrast, P. septica was only affected at the earlier time point. While the mechanisms contributing to nutrient shock are unclear, our data support the claim that certain Gram-negative bacteria may not be easily recovered on select nutrient rich agar.

\section{Conclusions}

To our knowledge, our modified culture methods are the first to consistently isolate Gram-negative bacterial commensals from human skin. Our culture methods yielded up to three species of CGN from a single individual and many individuals yielded just one species. It is likely that additional species not amenable to our culture methods exist and remain to be identified. Indeed, the microbiome literature on $16 \mathrm{~S}$ ribosomal DNA signatures suggests 2-5 classes of Gram-negative bacteria are present on the skin of a given individual [1, 15], although the number of different species represented within these classes is unknown. Consistent with our data, sophisticated analyses of metagenomic shotgun sequencing of the skin microbiome has identified Pseudomonas and Roseomonas species as well as other Gram-negative genera, although limitations in reference 
Table 1 Solid media type influences isolation of Gram-negative skin microbiota. (Upper) Growth results from initial swab collected from participant on various plates and at either 32 or $37^{\circ} \mathrm{C}$ using 4-quadrant streaking system. (Lower) Growth yields after inoculum isolated on R2A was re-plated under indicated conditions. Increasing amounts of bacterial growth are semi-quantitatively indicated as none (-), or as apparent only in the $1^{\text {st }}$ quadrant $(+)$, or into the $2^{\text {nd }}(++)$ or $3^{\text {rd }}(+++)$ streaked quadrant at $72 \mathrm{~h}$. Data are representative of three independent experiments for each species. Each species represented by 1-3 clinical isolates

\begin{tabular}{|c|c|c|c|c|c|c|c|c|c|c|}
\hline \multirow[b]{3}{*}{ Bacteria } & \multicolumn{10}{|c|}{ From Initial Swab } \\
\hline & \multicolumn{2}{|c|}{ Blood Agar } & \multicolumn{2}{|l|}{$\mathrm{BHI}$} & \multicolumn{2}{|c|}{ Chocolate } & \multicolumn{2}{|c|}{ MacConkey } & \multicolumn{2}{|l|}{$\mathrm{R} 2 \mathrm{~A}$} \\
\hline & 32 & 37 & 32 & 37 & 32 & 37 & 32 & 37 & 32 & 37 \\
\hline R. mucosa & - & - & - & - & - & - & - & - & +++ & + \\
\hline P. septica & - & - & - & - & - & - & - & - & +++ & + \\
\hline M. osloensis & - & - & - & - & - & - & - & - & +++ & + \\
\hline P. aeruginosa & ++ & - & - & - & ++ & + & ++ & + & +++ & + \\
\hline P. oryhabiztans & + & - & - & - & ++ & + & - & - & +++ & + \\
\hline \multirow[t]{3}{*}{ P. luteola } & + & - & - & - & ++ & + & - & - & +++ & + \\
\hline & \multicolumn{10}{|c|}{ After Isolation } \\
\hline & \multicolumn{2}{|c|}{ Blood Agar } & \multicolumn{2}{|l|}{$\mathrm{BHI}$} & \multicolumn{2}{|c|}{ Chocolate } & \multicolumn{2}{|c|}{ MacConkey } & R2A & \\
\hline Bacteria & 32 & 37 & 32 & 37 & 32 & 37 & 32 & 37 & 32 & 37 \\
\hline R. mucosa & ++ & ++ & + & + & - & - & - & - & +++ & ++ \\
\hline P. septica & ++ & ++ & ++ & ++ & ++ & ++ & + & + & +++ & ++ \\
\hline M. osloensis & ++ & ++ & ++ & ++ & + & + & - & - & +++ & ++ \\
\hline P. aeruginosa & ++ & - & ++ & ++ & ++ & + & + & ++ & +++ & ++ \\
\hline P. oryhabiztans & ++ & - & ++ & ++ & ++ & + & ++ & + & +++ & ++ \\
\hline P. luteola & ++ & ++ & ++ & ++ & ++ & + & ++ & + & +++ & ++ \\
\hline
\end{tabular}

genome databases and pooled analysis across individuals still prevented more exact determination of bacterial burden at the species level in a given individual [2]. Our data are also limited by the focus on forearm skin and exclusion of anaerobic culture conditions. For example, in a subset of patients with acne, $16 \mathrm{~S}$ ribosomal DNA data from facial swabs detected Escherichia coli [16], a bacterium we did not culture with our techniques. However, successful culture of even select Gram-negative microbiota using our new methodologies will allow whole genome sequencing and characterization of these specific colonizing strains at the biochemical, metabolic, and functional level that may lend insight into the interactions of these bacteria with their host.

\section{Ethics approval and consent to participate}

All clinical isolates were processed in a de-identified fashion under the National Institute of Allergy and Infectious Diseases Internal Review Board (IRB)-approved trial NCT02262819. All participants signed informed consent prior to isolate collection.

\section{Consent for publication}

Not applicable.

\section{Availability of data and material}

Supplemental information is available online. See Additional file 1 section below for details.

\section{Additional file}

Additional file 1: Figure S1. Growth of select commensal Gram-negative species after acute nutrient change. Data depicts the impact on growth for transferring cultures of $R$. mucosa, $P$. septica, $M$. osloensis from low nutrient broth to high nutrient broth. Supplemental Detailed Methods. Detailed protocol of Commensal Gram Negative Isolation. Step by step protocol used in CGN isolation from skin. (DOCX $707 \mathrm{~kb}$ )

\section{Abbreviations}

BHI: blood heart infusion; CGN: Commensal Gram-negative; MALDITOF: matrix-assisted laser desorption/ionization-time of flight; R2A: Reasoner's 2A; TSB: tryptic soy broth.

Competing interests

The authors declare they have no competing interests.

\section{Authors' contributions}

IAM developed the new protocol, performed some bacterial collections, and wrote the manuscript. KWW and JDR performed or assisted on bacterial collections and conducted agar culture and growth curve experiments. KMF conducted or supervised all MALDI-TOF analysis. IS performed coagulase testing. SKD oversaw the project and wrote the manuscript. All authors read and approved the final manuscript. 


\section{Acknowledgments}

We would like to acknowledge the participants for their assistance in this project. We thank Frida Stock, Julia Shah, and Elim Cho for technical assistance.

\section{Funding}

This work was supported by the Intramural Research Program of the National Institutes of Health and the National Institute of Allergy and Infectious Disease.

\section{Author details}

'Bacterial Pathogenesis Unit, Laboratory of Clinical Infectious Diseases, National Institute of Allergy and Infectious Diseases, National Institutes of Health, Bethesda, MD, USA. '2Department of Laboratory Medicine, National Institutes of Health Clinical Center, Bethesda, MD, USA.

Received: 25 November 2015 Accepted: 29 March 2016

Published online: 06 April 2016

\section{References}

1. Grice EA, Kong HH, Conlan S, Deming CB, Davis J, Young AC, Program NCS, Bouffard GG, Blakesley RW, Murray PR, et al. Topographical and temporal diversity of the human skin microbiome. Science. 2009;324(5931):1190-2.

2. Oh J, Byrd AL, Deming C, Conlan S, Program NCS, Kong HH, Segre JA. Biogeography and individuality shape function in the human skin metagenome. Nature. 2014:514(7520):59-64.

3. Marples MJ. The normal flora of the human skin. Br J Dermatol. 1969;81 Suppl 1:2-13.

4. Lowbury EJ. Gram-negative bacilli on the skin. Br J Dermatol. 1969;81 Suppl 1:55+.

5. Conlan S, Kong HH, Segre JA. Species-level analysis of DNA sequence data from the NIH Human Microbiome Project. PLoS One. 2012;7(10):e47075.

6. Sandle T. An approach for the reporting of microbiological results from water systems. PDA J Pharm Sci Technol. 2004;58(4):231-7.

7. Lau AF, Wang H, Weingarten RA, Drake SK, Suffredini AF, Garfield MK, Chen Y, Gucek M, Youn JH, Stock F, et al. A rapid matrix-assisted laser desorption ionization-time of flight mass spectrometry-based method for single-plasmid tracking in an outbreak of carbapenem-resistant Enterobacteriaceae. J Clin Microbiol. 2014;52(8):2804-12.

8. Lau AF, Drake SK, Calhoun LB, Henderson CM, Zelazny AM. Development of a clinically comprehensive database and a simple procedure for identification of molds from solid media by matrix-assisted laser desorption ionization-time of flight mass spectrometry. J Clin Microbiol. 2013;51(3):828-34.

9. Youn JH, Drake SK, Weingarten RA, Frank KM, Dekker JP, Lau AF. Clinical Performance of a Matrix-Assisted Laser Desorption Ionization Time-of-Flight Mass Spectrometry Method for the Detection of Certain blaKPC-containing Plasmids. J Clin Microbiol. 2016;54(1):35-42.

10. Stevenson LG, Drake SK, Shea YR, Zelazny AM, Murray PR. Evaluation of matrix-assisted laser desorption ionization-time of flight mass spectrometry for identification of clinically important yeast species. J Clin Microbiol. 2010;48(10):3482-6.

11. Saleeb PG, Drake SK, Murray PR, Zelazny AM. Identification of mycobacteria in solid-culture media by matrix-assisted laser desorption ionization-time of flight mass spectrometry. J Clin Microbiol. 2011;49(5):1790-4.

12. Wauters G, Vaneechoutte M. Chapter 33: Approaches to the Identification of AerobicGram-Negative Bacteria. In: Jorgensen J, Pfaller M, Carroll K, Funke G, Landry M, Richter S, Warnock D, editors. Manual of Clinical Microbiology. Eleventh Edition. Washington: ASM Press; 2015. p. 613-634.

13. Liu Z, Wang L, Luo Z, Heusch Al, Cascioli V, McCarthy PW. Microenvironment temperature prediction between body and seat interface using autoregressive data-driven model. J Tissue Viability. 2015;24(4):131-9.

14. Azevedo NF, Braganca SM, Simoes LC, Cerqueira L, Almeida C, Keevil CW, Vieira MJ. Proposal for a method to estimate nutrient shock effects in bacteria. BMC Res Notes. 2012;5:422.

15. Kong HH, Oh J, Deming C, Conlan S, Grice EA, Beatson MA, Nomicos E, Polley EC, Komarow HD, Program NCS, et al. Temporal shifts in the skin microbiome associated with disease flares and treatment in children with atopic dermatitis. Genome Res. 2012;22(5):850-9.

16. Fitz-Gibbon S, Tomida S, Chiu BH, Nguyen L, Du C, Liu M, Elashoff D, Erfe MC, Loncaric A, Kim J, et al. Propionibacterium acnes strain populations in the human skin microbiome associated with acne. J Invest Dermatol. 2013;133(9):2152-60.

\section{Submit your next manuscript to BioMed Central and we will help you at every step:}

- We accept pre-submission inquiries

- Our selector tool helps you to find the most relevant journal

- We provide round the clock customer support

- Convenient online submission

- Thorough peer review

- Inclusion in PubMed and all major indexing services

- Maximum visibility for your research

Submit your manuscript at www.biomedcentral.com/submit
Biomed Central 